\title{
TRAP1 regulates stemness through Wnt/ $\beta$-catenin pathway in human colorectal carcinoma
}

\author{
Giacomo Lettini ${ }^{1}$, Lorenza Sisinni ${ }^{1}$, Valentina Condelli ${ }^{1}$, Danilo Swann Matassa ${ }^{2}$, Vittorio Simeon ${ }^{1}$, Francesca Maddalena ${ }^{1}$, \\ Marica Gemei ${ }^{3}$, Elvira Lopes ${ }^{1}$, Giulia Vita ${ }^{4}$, Luigi Del Vecchio ${ }^{3}$, Franca Esposito ${ }^{\star, 2}$ and Matteo Landriscina ${ }^{* 1,5}$
}

\begin{abstract}
Colorectal carcinoma (CRC) is a common cause of cancer-related death worldwide. Indeed, treatment failures are triggered by cancer stem cells (CSCs) that give rise to tumor repopulation upon initial remission. Thus, the role of the heat shock protein TRAP1 in stemness was investigated in CRC cell lines and human specimens, based on its involvement in colorectal carcinogenesis, through regulation of apoptosis, protein homeostasis and bioenergetics. Strikingly, co-expression between TRAP1 and stem cell markers was observed in stem cells located at the bottom of intestinal crypts and in CSCs sorted from CRC cell lines. Noteworthy, TRAP1 knockdown reduced the expression of stem cell markers and impaired colony formation, being the CSC phenotype and the anchorage-independent growth conserved in TRAP1-rich cancer cells. Consistently, the gene expression profiling of HCT116 cells showed that TRAP1 silencing results in the loss of the stem-like signature with acquisition of a more-differentiated phenotype and the downregulation of genes encoding for activating ligands and target proteins of Wnt/ $\beta$-catenin pathway. Mechanistically, TRAP1 maintenance of stemness is mediated by the regulation of $\mathrm{Wnt} / \beta$-catenin signaling, through the modulation of the expression of frizzled receptor ligands and the control of $\beta$-catenin ubiquitination/phosphorylation. Remarkably, TRAP1 is associated with higher expression of $\beta$-catenin and several Wnt $/ \beta$-catenin target genes in human CRCs, thus supporting the relevance of TRAP1 regulation of $\beta$-catenin in human pathology. This study is the first demonstration that TRAP1 regulates stemness and Wnt $/ \beta$-catenin pathway in CRC and provides novel landmarks in cancer biology and therapeutics.
\end{abstract}

Cell Death and Differentiation (2016) 23, 1792-1803; doi:10.1038/cdd.2016.67; published online 23 September 2016

Colorectal carcinoma (CRC) represents $\sim 10 \%$ of the global incidence of cancer, ${ }^{1}$ being the second cause of cancer death in the United States and Europe. ${ }^{2}$ Despite significant improvements in treatment strategies, its prognosis remains poor and the main cause of treatment failure is drug resistance. ${ }^{3}$ Thus, novel molecular targets are needed to improve patient outcome. In such a context, main attention has been focused on the role of cancer stem cells (CSCs), a subpopulation of cancer cells with the exclusive ability to self-renew and drive tumor growth. Indeed, CSCs are organized in a cellular hierarchy with a pool of stem cells on the top of an organized system from which tumor cells originate. CSCs are the only ones with (i) selfrenewal ability, causing the initiation and the maintenance of the tumor and (ii) the capacity to reversibly differentiate into heterogeneous non-tumorigenic cancer cell types that constitute the bulk of the tumor. ${ }^{4}$ In recent years, the CSC theory led to the notion that CSCs are resistant to apoptosis and, thus, not eradicated by current treatments and responsible for tumor recurrence. ${ }^{5}$ Indeed, CSCs are functionally protected in a tissue stem cell niche where they are quiescent but viable, and therefore prepared to regenerate the tumor, even after long time and distant from the primary site. The stem niche is a hypoxic functional compartment, that induces a metabolic reprogramming of CSCs toward glycolysis ${ }^{6}$ and favors the expression of genes involved in protection from apoptosis, ${ }^{7}$ allowing them to survive in a hostile environment and escape from anticancer therapies. ${ }^{7}$

TRAP1 is a component of the HSP90 chaperone family, with a prevalent mitochondrial localization, initially characterized for its capacity to protect against oxidative stress and favor mitochondria integrity ${ }^{8}$ and subsequently involved in several other cellular processes critical for colorectal carcinogenesis. ${ }^{9}$ In fact, TRAP1 (i) belongs to a pro-survival signaling pathway responsible for protection from apoptosis and drug resistance; ${ }^{9-12}$ (ii) contributes to mitochondrial bioenergetics, favoring a context-dependent reprogramming of oxidative metabolism; ${ }^{13-15}$ (iii) controls proteostasis by a co-translational regulation of protein synthesis/ubiquitination. ${ }^{11,16-18}$ It is worth

${ }^{1}$ Laboratory of Pre-Clinical and Translational Research, IRCCS, Referral Cancer Center of Basilicata, Rionero in Vulture, PZ, Italy; ${ }^{2}$ Department of Molecular Medicine and Medical Biotechnology, University of Naples Federico II, Naples, Italy; ${ }^{3} \mathrm{CEINGE}$, Biotecnologie Avanzate, University of Naples Federico II, Naples, Italy; ${ }^{4}$ Pathology Unit, IRCCS, Referral Cancer Center of Basilicata, Rionero in Vulture, PZ, Italy and ${ }^{5}$ Medical Oncology Unit, Department of Medical and Surgical Sciences, University of Foggia, Foggia, Italy

${ }^{*}$ Corresponding author: F Esposito, Dipartimento di Medicina Molecolare e Biotecnologie Mediche, Università degli Studi di Napoli Federico II, Via S. Pansini 5, Napoli 80131, Italy. Tel: +39 081 7463145; Fax: +39 0817464359.

or M Landriscina, Dipartimento di Scienze Mediche e Chirurgiche, Università degli Studi di Foggia, Viale Pinto, 1, Foggia 71100 , Italy. Tel: +39 0881736426 ; Fax: +39 0972726482.

E-mail: franca.esposito@unina.it or matteo.landriscina@unifg.it

Abbreviations: CRC, colorectal carcinoma; CSC, cancer stem cell; TRAP1, TNF receptor-associated protein 1; TA, transit-amplifying; FACS, fluorescence-activated cell sorting; co-ip, co-immunoprecipitation; ER, endoplasmic reticulum; ATCC, American Type Culture Collection; STR, short tandem repeat; FBS, fetal bovine serum; PBS, phosphate buffer saline; PE, phycoerythrin; APC, allophycocyanin; FITC, fluorescein isothiocyanate; MFI, mean fluorescence intensity; DAPI, 4',6-diamidin-2-fenilindolo Received 19.2.16; revised 30.5.16; accepted 08.6.16; Edited by Y Shi; published online 23.9.2016 
a

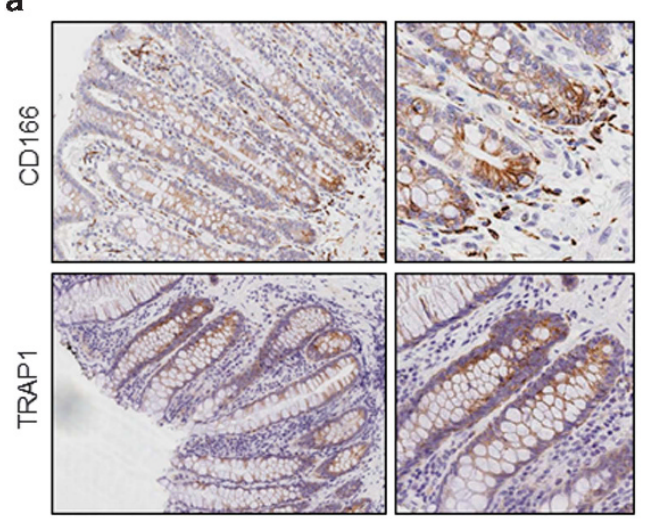

b

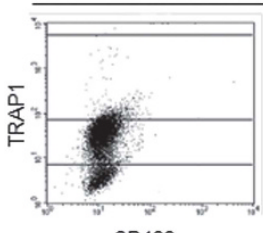

CD133
HCT116 cells

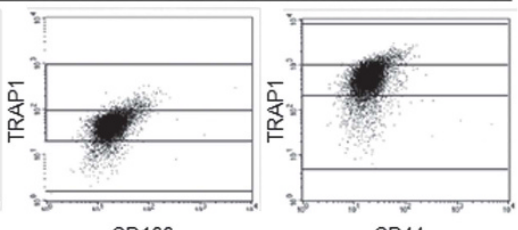

CD166

$\mathrm{CD} 44$ c

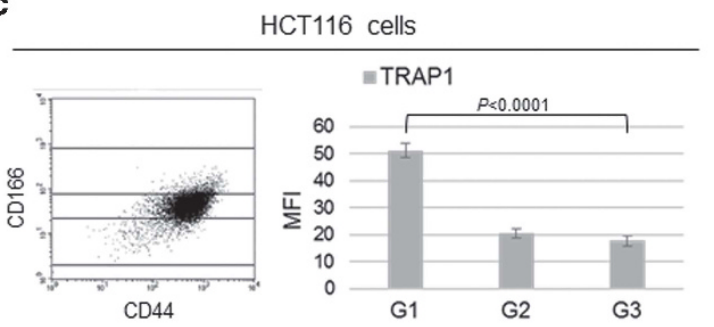

d
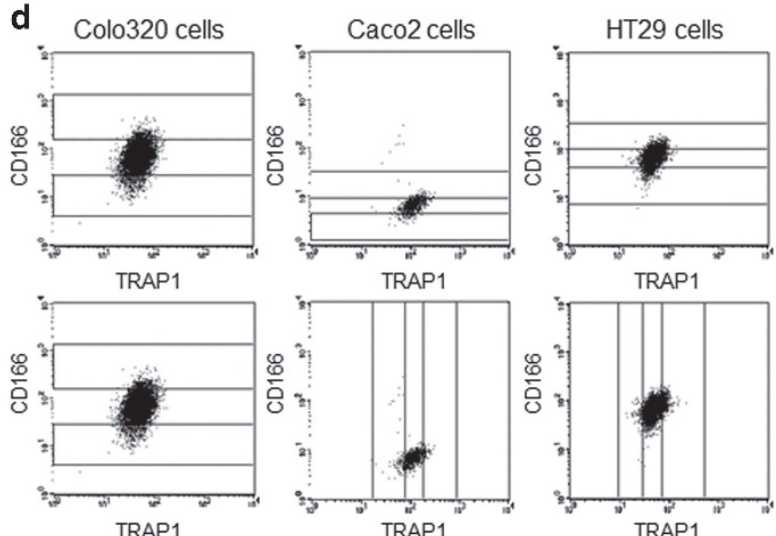

Figure 1 TRAP1 expression is enriched in stem cells of human colon mucosa and colorectal carcinoma cell lines. (a) Immunohistochemical analysis of TRAP1 and CD166 expression in normal colonic mucosa (original magnification, $\times 10$ ). (b) Cytometric dot plot reporting CD133, CD166 and CD44 expression versus TRAP1 expression in HCT116 cells. (c) Cytometric dot plot (left panel) reporting CD44 expression versus CD166 expression in HCT116 cells and bar graph (right panel) reporting TRAP1 mean fluorescence intensity (MFI) in three-gated subpopulations with increasing levels of both CD166 and CD44. P-values represent the statistical significance of TRAP1 expression levels according to the gating strategy. Data represent mean + S.D. (d) Cytometric dot plot reporting CD166 expression versus TRAP1 expression in COLO320, CaCo2 and HT29 cells

noting that TRAP1 is upregulated in several human malignancies and, among others, CRC and its levels correlate with a drug-resistant phenotype, ${ }^{8,10}$ extensive lymph node dissemination and poor patient outcome. ${ }^{19}$

Therefore, given the complexity of the molecular mechanisms that control the biology of CSCs and the well-established role of TRAP1 in regulating the plasticity of cancer cells, ${ }^{9}$ this study was designed to address the hypothesis that TRAP1 is involved in maintenance of stemness in CRC cells. Here, we report, for the first time, that TRAP1 regulates the CSC phenotype by modulating $\mathrm{Wnt} / \beta$-catenin pathway.

\section{Results}

TRAP1 expression is enriched in stem cells of human colon mucosa and CRC cell lines. TRAP1 is expressed, although at low levels, in human normal colonic mucosa ${ }^{10,20}$ and intestinal stem cells are located in the basal portion of the crypts; ${ }^{21}$ thus, TRAP1 and the stem cell marker CD166, widely expressed in colon stem cells, ${ }^{22}$ were comparatively evaluated in normal colonic mucosa. Interestingly, TRAP1 is coexpressed with CD166 in cells located in the basal portion of the crypts, whereas its expression is progressively reduced from the transit-amplifying (TA) zone to the lumen (Figure 1a). As TRAP1 is highly expressed in human CRCs and CRC cell lines, ${ }^{10}$ the co-expression of TRAP1 and CSC surface markers was further evaluated in human $\mathrm{CRC}$ cells by multidimensional flow cytometry. To this purpose, we selected a panel of surface CSC antigens, widely used for the phenotypic characterization of human colorectal CSCs, that is, CD133, CD166 and CD44. ${ }^{23}$ Interestingly, a striking correlation was observed between the expression of all stem cell markers analyzed and TRAP1 levels in HCT116 cells (Figure 1b). In order to analyze the expression of TRAP1 in CSCs and its co-expression with CSC markers, three subpopulations with increasing levels of CD133, CD166 or CD44 were selected and a progressive increase in TRAP1 expression was observed according to the upregulation of CSC markers, being TRAP1 expression significantly higher in CD133-, CD166- and CD44-bright cell subpopulations (Supplementary Figure S1A). Of note, TRAP1 expression was also enriched in the CD166/CD44-double positive HCT116 cell subpopulation (Figure 1c). A similar coexpression was observed between CD166 and TRAP1 in CRC COLO320, CaCo2 and HT29 cells (Figure 1d) and, consistently, TRAP1 expression was significantly and constantly higher in CD166-bright cell subpopulations (Supplementary Figure S1B).

Among different available CRC cell lines, HCT116 cells were selected for further studies, as they are characterized by a stem-like phenotype with constitutive activation of Wnt/ $\beta$ catenin pathway, a key player in colon CSCs self-renewal. ${ }^{24}$ Thus, HCT116 cells were sorted according to CD166 expression and CD166-bright and CD166-low cell subpopulations were isolated. As expected, the expression of the stem cell markers CD166 and CD44 was highly enriched in CD166bright (right panels) compared with CD166-low (left panels) HCT116 cells (Figure 2a). Interestingly, immunoblot analysis of sorted cell subpopulations showed higher expression of TRAP1 in CD166-bright cells (Figure 2b). In parallel 
a
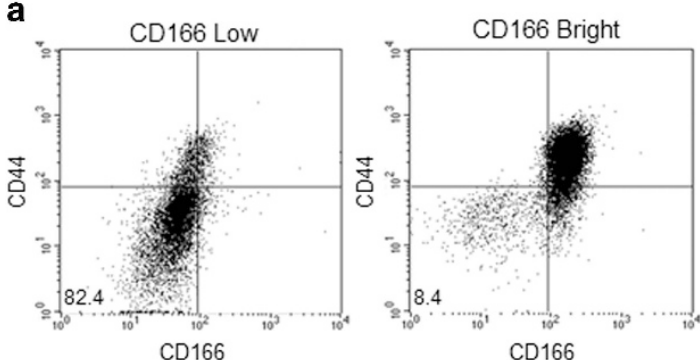

C

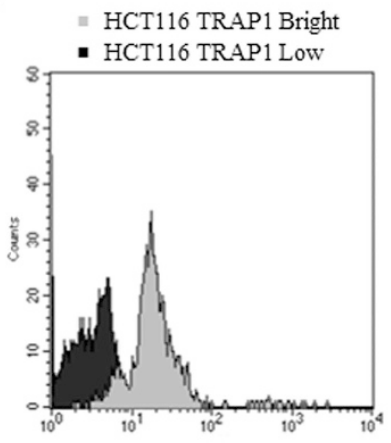

b

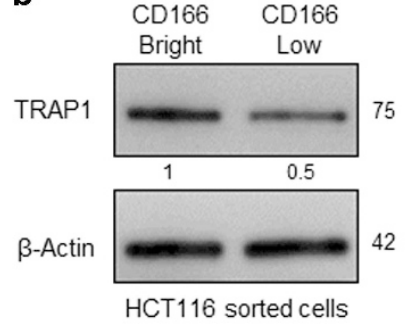

d
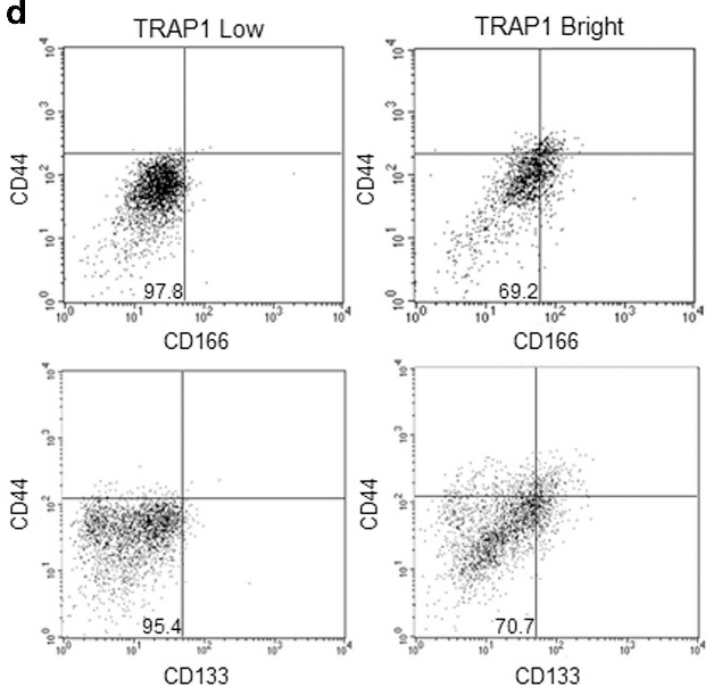

Figure 2 TRAP1 is upregulated in CD166-bright cell subpopulation sorted from colon carcinoma cells. (a) Cytometric dot plot reporting CD166 and CD44 expression levels in sorted CD166-bright and CD166-low HCT116 cell subpopulations. Percentages of cells in the lower left quadrant are reported. (b) Total cell lysates from sorted CD166-bright and CD166-low HCT116 cell subpopulations were separated by SDS-PAGE and immunoblotted with the indicated antibodies. (c) TRAP1 expression levels in sorted TRAP1-low and TRAP1-bright HCT116 cell subpopulations. (d) Cytometric dot plot reporting CD166, CD133 and CD44 expression levels in sorted TRAP1-bright and TRAP1-low HCT116 cell subpopulations. Percentages of cells in the lower left quadrant are reported

experiments, HCT116 cells were sorted according to TRAP1 expression and this resulted in two distinct cell subpopulations, that is, TRAP1-low and TRAP1-bright cells (Figure 2c). Of note, FACS analysis showed an enrichment in CD44, CD166 and CD133 expression in TRAP1-bright HCT116 cells (Figure 2d). These data suggest that TRAP1 is upregulated in colon CSCs, being expressed at lower level in the remaining tumor cell population.

TRAP1 modulates the clonogenic ability of colon carcinoma cells. The co-expression between TRAP1 and CD166 was further evaluated in TRAP1 stably interfered HCT116 cells in comparison with scramble cells (Figure $3 a$ ) and in three cell subpopulations with increasing TRAP1 levels gated from both scramble and shTRAP1 HCT116 cells (Figure $3 b$ ). Noteworthy, the downregulation of TRAP1 expression (Figure 3a, insert) resulted in lower CD166 expression in the whole cell population (Figure 3a), and CD166 expression paralleled TRAP1 levels in selected subpopulations, being significantly higher in TRAP1-bright cells from scramble HCT116 cells (Figure 3b). In further experiments, we questioned whether TRAP1 modulates the anchorageindependent growth of HCT116 cells. Thus, scramble and shTRAP1 HCT116 cells were compared for their clonogenic ability, and, noteworthy, TRAP1 knocking down resulted in a significant impairment in colony formation (Figure $3 \mathrm{c}$ ), with a $1: 4$ ratio in colony numbers between shTRAP1 and scramble HCT116 cells (Figure 3d). Intriguingly, TRAP1 expression was re-evaluated in the few colonies collected from shTRAP1 HCT116 cells in comparison with colonies obtained from scramble cells and no difference was observed in TRAP1 levels (Figure $3 e$ ). Consistently, HCT116 cells obtained from colonies were subdivided in three cell subpopulations with increasing TRAP1 levels and, differently from originating cell lines (Figure 3b), no difference in TRAP1 and CD166 expression was observed between scramble and shTRAP1 cells (Figure 3f).

In subsequent experiments, we evaluated whether the upregulation of TRAP1 restores the stem cell phenotype in shTRAP1 HCT116 cells. HCT116 shTRAP1 cells were transfected with pMock or TRAP1 cDNA (Figure 4a, insert) and the co-expression between TRAP1 and CD166 was evaluated in the whole population (Figure $4 \mathrm{a}$ ) and in subpopulations with increasing TRAP1 levels (Figure 4b). Interestingly, TRAP1 transfection rescued the CD166-low phenotype in shTRAP1 HCT116 cells (Figure 4a) and, consistently, CD166 expression was maximal in TRAP1-bright cell subpopulation obtained upon TRAP1 re-transfection (Figure 4b). These data 
a
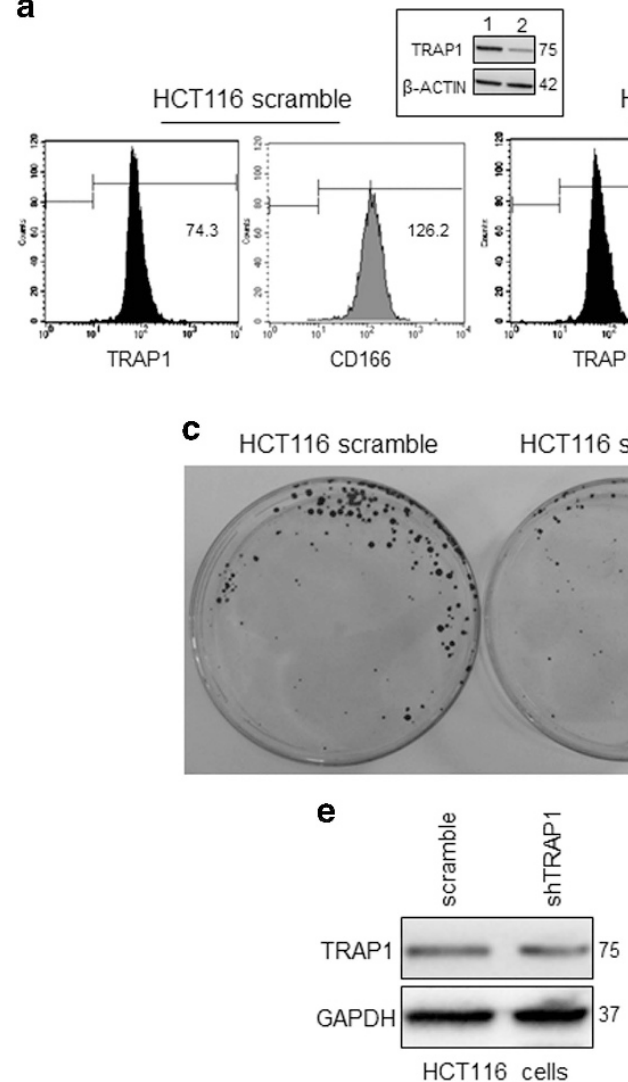

b

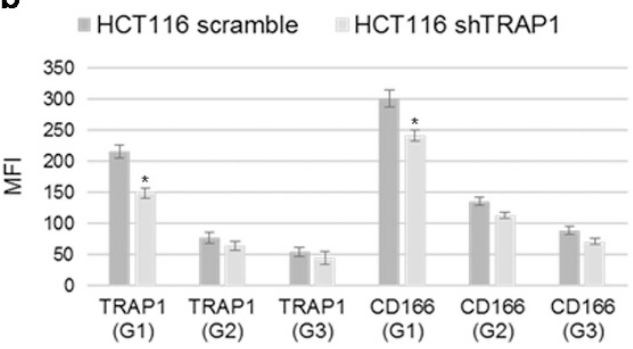

d

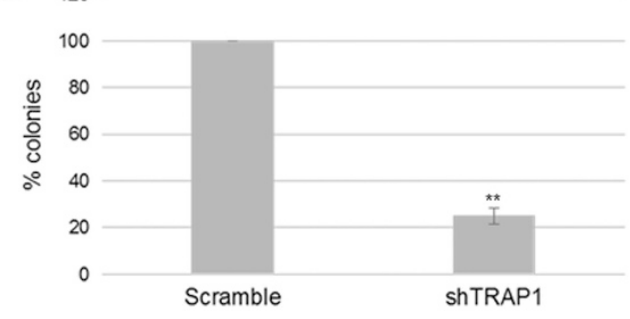

f

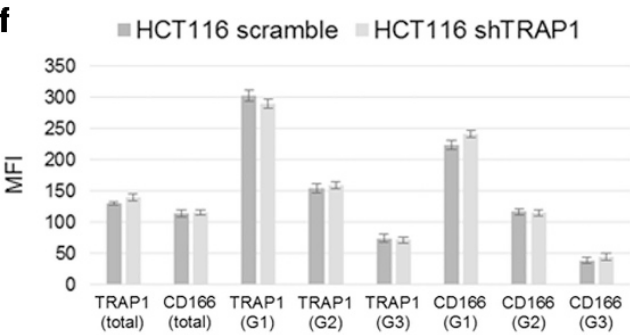

Figure 3 TRAP1 modulates the clonogenic ability of colon carcinoma cells. (a) TRAP1 and CD166 expression in scramble and shTRAP1 HCT116 cells. Percentages reported in flow plots indicate the MFI of total populations. Insert: TRAP1 immunoblot analysis in scramble (1) and shTRAP1 (2) HCT116 cells. (b) Relative TRAP1/CD166 MFIs in three-gated subpopulations with increasing TRAP1 levels obtained from scramble and shTRAP1 HCT116 cells. Statistical significance compared with the respective scramble control: ${ }^{*} P<0.0001$. Data represent mean + S.D. (c, d) Representative culture plates (c) and quantitative representation (d) of the clonogenic potential of scramble and shTRAP1 HCT116 cells. Statistical significance compared with scramble cells: ${ }^{* *} P<0.001$. Data represent mean + S.D. (e) Total cell lysates from colonies collected from scramble and shTRAP1 HCT116 cells were separated by SDS-PAGE and immunoblotted with the indicated antibodies. (f) Relative TRAP1/CD166 MFIs in total population and gated subpopulations with increasing TRAP1 levels obtained from scramble and shTRAP1 colonies. Data represent mean + S.D.

suggest that TRAP1 and CSC markers are co-expressed in CSCs and TRAP1 is responsible for the clonogenic ability of CRC cells.

TRAP1 silencing results in the loss of the stem-like phenotype and the downregulation of $\mathrm{Wnt} / \mathrm{\beta}$-catenin pathway. As a recent study of gene expression classification of human CRCs associated HCT116 cells to the stem-like subtype, based on the constitutive activation of $\mathrm{Wnt} / \beta$-catenin signaling, ${ }^{24,25}$ we questioned whether TRAP1 silencing was able to reprogram this gene signature. To address this issue we took advantage from our recently published wholegenome gene expression profiling of TRAP1-silenced HCT116 cells. ${ }^{26}$ Thus, the concordance in the gene expression profiles between shTRAP1 HCT116 cells and the gene signatures representative of the five different CRC subtypes ${ }^{24}$ was analyzed. Noteworthy, TRAP1 silencing resulted in loss of the stem-like phenotype, being the shTRAP1 signature discordant in $66.2 \%$ of genes compared with the stem-like signature (Supplementary Tables S1 and S2). Indeed, shTRAP1 HCT116 cells showed a higher degree of concordance with the more-differentiated Enterocyte subtype signature (Supplementary Tables S1 and S2). Consistently, unsupervised analysis with Euclidean dendrogram showed higher proximity of shTRAP1 HCT116 cell to Globet-like and Enterocyte subtypes (Figure 4c). Furthermore, the analysis of our data set showed that TRAP1 silencing induced the reversion of the expression of specific genes responsible for the stem-like signature (Figure 4d), most of them encoding for ligands of the frizzled family receptors responsible for activation of Wnt signaling ${ }^{27}$ or target proteins of $\mathrm{Wnt} / \beta$ catenin pathway. ${ }^{28}$

Thus, to investigate further the mechanism responsible for TRAP1 regulation of stemness, we focused our attention on Wnt/ $\beta$-catenin pathway, which regulates colon CSCs selfrenewal. ${ }^{27}$ In preliminary experiments, the expressions of some of these TRAP1-modulated Wnt-related genes (i.e., WNT3A, WNT16, c-JUN, c-MYC, CCND1, AXIN2, SP5, NKD1) ${ }^{28}$ was analyzed by quantitative PCR in TRAP1-silenced HCT116 and HT29 (Figure 5a) cells; indeed, these genes were significantly downregulated in both TRAP1-silenced CRC cell lines (Figure $5 \mathrm{a}$ ). The regulation of $\mathrm{Wnt} / \beta$-catenin signaling by TRAP1 was further evaluated, by immunoblot analysis, in different $C R C$ cell lines and the downregulation of WNT3A was confirmed in TRAP1-silenced HCT116 and Caco2 cells, as well as BIRC5 (SURVIVIN) was found downregulated in HCT116, 
a

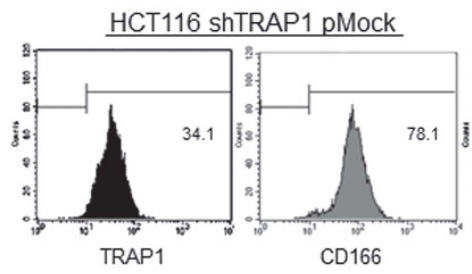

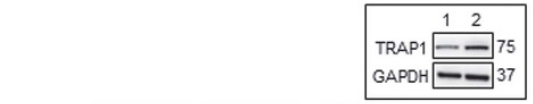
HCT116 ShTRAP1 PTRAP1

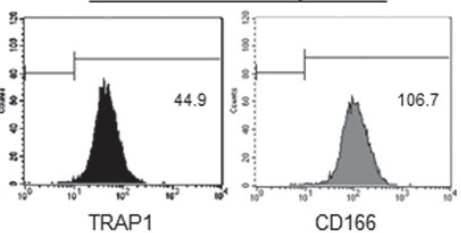

b

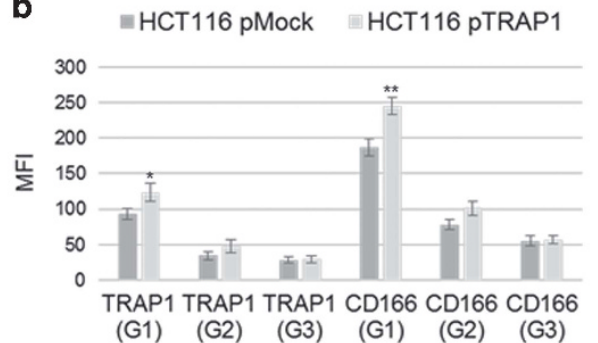

c
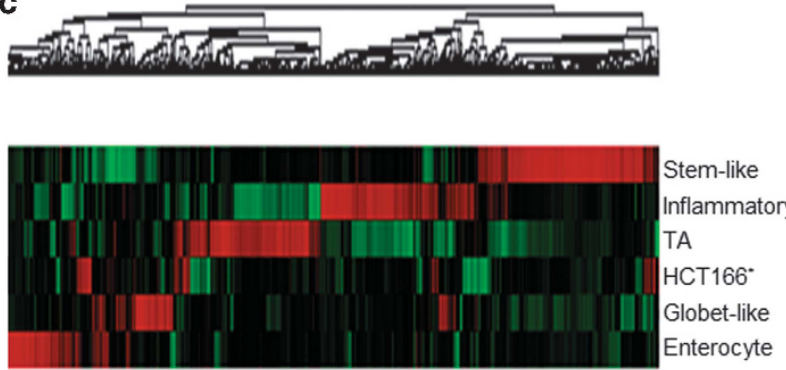

d

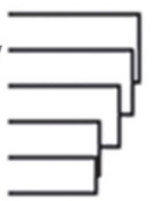

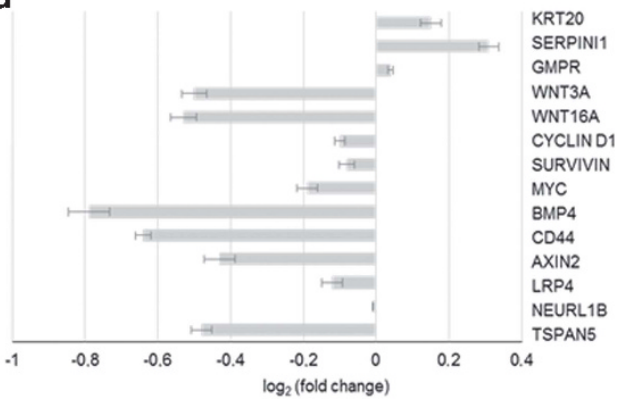

Figure 4 TRAP1 is responsible for the Stem-like phenotype of colon carcinoma cells. (a) TRAP1 and CD166 expression in Mock-and TRAP1-transfected shTRAP1 HCT116 cells. Values reported in flow plots indicate the percentages of MFI in the whole HCT116 cell population. Insert: TRAP1 immunoblot analysis in shTRAP1 HCT116 cells transfected with pMock (1) or pTRAP1 (2) expression vectors. (b) Relative TRAP1/CD166 MFIs in three-gated subpopulations with increasing TRAP1 levels obtained from Mockand TRAP1-transfected shTRAP1 HCT116 cells. Statistical significance compared to the respective pMock control: ${ }^{*} P<0.01 ;{ }^{* *} P<0.0001$. Data represent mean + S.D. (c) Heat map and unsupervised analysis of shTRAP1 HCT116 cells and five CRC subtype signatures. Euclidean clustering shows distances and grouping of signatures. (d) Gene expression analysis of selected genes responsible for the Stem-like phenotype of HCT116 cells. Bar graphs report the $\log _{2}$ (fold change) for each gene expression in shTRAP1 HCT116 cells respect to scramble cells. Data represent mean + S.D.

HT29 and CaCo2 cells under TRAP1 silencing conditions (Figures $5 \mathrm{~b}$ and $\mathrm{c}$ ). Notably, WNT3A was undetectable both at mRNA and protein levels in HT29 and COLO320 cells. As these genes encode for ligands (i.e., WNT3A and WNT16) of the frizzled family receptors ${ }^{27}$ or $\mathrm{Wnt} / \beta$-catenin signaling target genes (i.e., c-JUN, c-MYC, CCND1, AXIN2, SP5, NKD1), ${ }^{28}$ all together these data support the hypothesis that TRAP1 modulates the expression of components of $\mathrm{Wnt} / \beta$-catenin pathway, and its silencing results in attenuation of $\mathrm{Wnt} / \beta$ catenin signaling.

TRAP1 regulates $\beta$-catenin ubiquitination/phosphorylation. We further evaluated TRAP1 regulation on $\beta$-catenin, a subunit of the cadherin protein complex: $\beta$-catenin is an intracellular signal transducer in Wnt signaling pathway, being able to translocate to the nucleus and induce the transcription of several target genes. ${ }^{29}$ The cellular level of $\beta$-catenin is mostly controlled by its phosphorylation, which drives the ubiquitination and the proteosomal degradation of the protein; whereas the activation of Wnt pathway results in increase of non-phosphorylated $\beta$-catenin active form, thus enabling its transcriptional activity. ${ }^{27}$ Indeed, although mRNA levels of CTNNB1 ( $\beta$-catenin) were unchanged in TRAP1-silenced cells (Figure $5 \mathrm{a}$ ), a reduced expression of the active form of $\beta$-catenin and, with less extent, of the whole protein was observed in CRC cell lines (Figures $5 \mathrm{~b}$ and $\mathrm{c}$ ).

The hypothesis that TRAP1 regulates $\beta$-catenin ubiquitination/phosphorylation was further explored. Indeed, TRAP1 regulates the quality of its client proteins, ${ }^{20,26}$ being their expression levels higher in a TRAP1-rich background, whereas their ubiquitination increased upon disruption of TRAP1 quality-control network. ${ }^{16,17,20,26}$ This TRAP1 function is mediated by its interaction with the proteasome regulatory particle TBP7; ${ }^{16}$ thus, in preliminary experiments we questioned whether $\beta$-catenin levels were downregulated upon TBP7 interference, as observed under TRAP1 silencing condition. Indeed, the active form of $\beta$-catenin was downregulated upon TBP7 silencing (Figure 6a), this suggesting that its degradation is controlled by TRAP1/TBP7 network. In parallel experiments, we questioned whether the reduced protein levels of the active form of $\beta$-catenin observed in TRAP1- and TBP7-interfered cells (Figures $5 \mathrm{~b}$ and $6 \mathrm{a}$ ) were dependent on increased phosphorylation and ubiquitination. Thus, $\beta$-catenin was immunoprecipitated from HCT116 cells upon TRAP1 or TBP7 transient silencing and immunoblot analysis of $\beta$-catenin immunoprecipitates showed increase of its ubiquitination and phosphorylation in TRAP1- and TBP7interfered cells (Figure 6b). Finally, $\beta$-catenin ubiquitination/ phosphorylation levels were evaluated upon transfection of a TBP7 deletion mutant unable to bind TRAP1, which behaves as dominant negative over the TRAP1/TBP7 quality-control. ${ }^{16}$ Consistently, the disruption of TRAP1/TBP7 network resulted in increased ubiquitination and phosphorylation of $\beta$-catenin (Figure 6c). Altogether, these observations suggest that TRAP1/TBP7 regulation of $\beta$-catenin ubiquitination occurs concurrently with its phosphorylation and that the suppression 


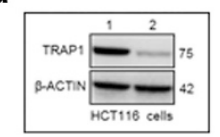

"HCT116 cells $=$ HT29 cells

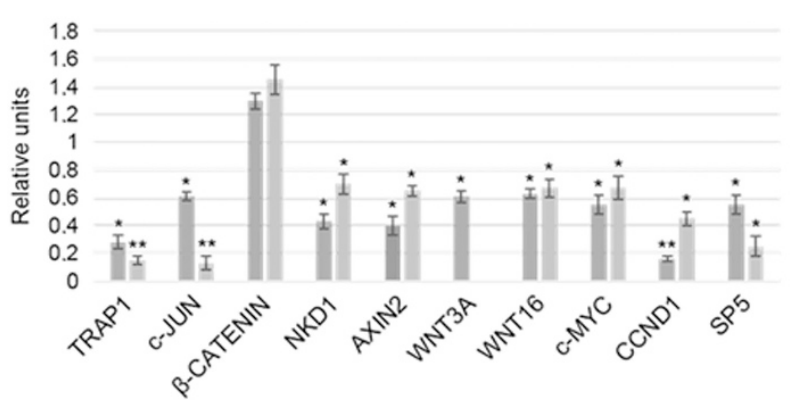

C

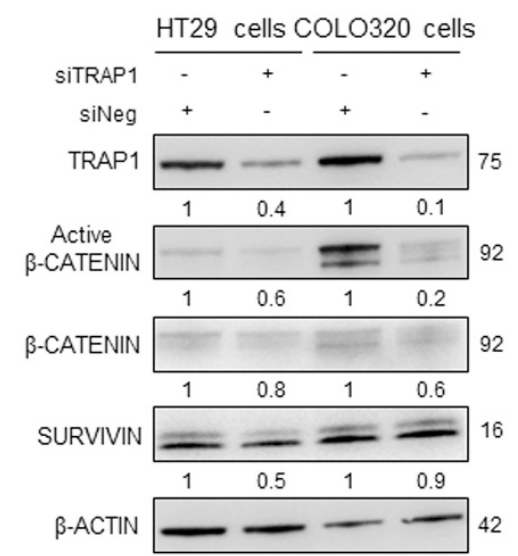

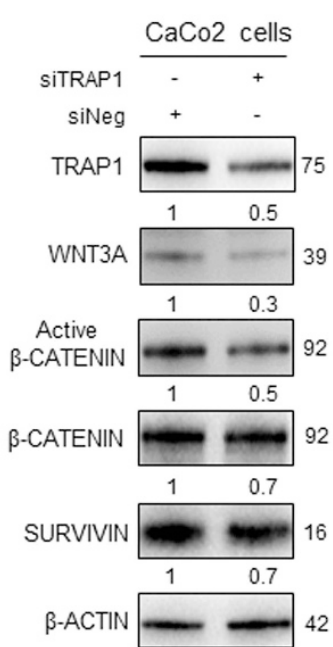

b

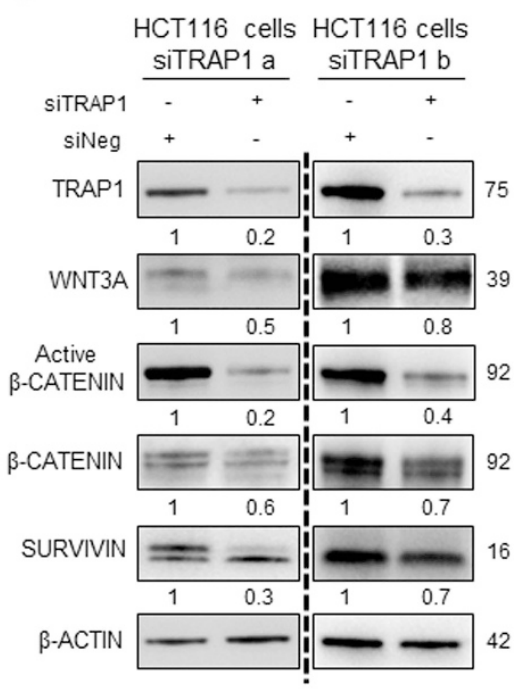

Figure 5 TRAP1 regulates Wnt/ $\beta$-catenin pathway in colon carcinoma cells. (a) Real-time PCR gene expression analysis of selected genes in TRAP1-silenced HCT116 and HT29 cells compared with cells transfected with negative siRNA: ${ }^{*} P<0.01 ;{ }^{*} P<0.001$. Inserts: TRAP1 immunoblot analysis in control (1) and TRAP1-silenced (2) HCT116 and HT29 cells. Data represent mean + S.D. (b, c) TRAP1, Wnt3A, $\beta$-catenin and survivin immunoblot analysis in total cell lysates from HCT116 cells transfected with control and two TRAP1 independent siRNAs (b) and HT29, COLO320 and CaCo2 cells transfected with control or TRAP1 siRNAs (c)

of TRAP1 network results in increased $\beta$-catenin phosphorylation and ubiquitination. As only the non-phosphorylated active form of $\beta$-catenin migrates to the nucleus acting as a transcription factor, ${ }^{29}$ we questioned whether TRAP1 regulation of $\beta$-catenin yields reduced nuclear levels. Consistently with the reduced expression of several $\beta$-catenin target genes, TRAP1 silencing resulted in lower levels of the active form and the whole protein in the nuclear fraction of HCT116 cells (Figure 6d). Taken as a whole, these observations suggest that $\beta$-catenin is among the genes whose ubiquitination is enhanced in a TRAP1-low background, being its expression higher in condition of TRAP1 upregulation.

$\boldsymbol{\beta}$-catenin interacts with TRAP1 network proteins. The hypothesis that TRAP1 and TBP7 interact with $\beta$-catenin was evaluated by co-immunoprecipitation (co-ip) experiments (Figures $7 a$ and b). Indeed, $\beta$-catenin immunoblot analysis of TRAP1 (Figure 7a) and TBP7 (Figure 7b) co-immunoprecipitates detected a $90 \mathrm{kDa}$ band, immunoreactive with $\beta$-catenin antibody. In parallel experiments, confocal microscopy and in situ Proximity Ligation assay were used to establish whether TRAP1 and $\beta$-catenin colocalize and are components of an intracellular protein complex. Indeed, double immunofluorescence staining showed that $\beta$-catenin co-distributes to a great extent with TRAP1 in HCT116 cells (Figure 7c) and direct proteinprotein interaction was observed between TRAP1 and $\beta$ catenin upon in situ Proximity ligation assay (Figure 7d). The specificity of these interactions was confirmed upon TRAP1 or TBP7 interference in HCT116 cells: of note, a significant reduction of $\beta$-catenin co-ip was observed in parallel with TRAP1 or TBP7 downregulation (Figure 7e).

As our data suggest that TRAP1 regulates $\beta$-catenin levels and stemness, COLO320 cells were transfected with TRAP1 and evaluated for the expression of $\beta$-catenin (Figure 8a, left panel) and stem cell markers (Figure $8 \mathrm{a}$, right panel). It is noteworthy that TRAP1 upregulation resulted in a parallel upregulation of $\beta$-catenin and CD166 and CD44 levels (Figure 8a). These evidences suggest that TRAP1 and $\beta$-catenin are components of a multiprotein complex and that TRAP1 modulates stemness through $\beta$-catenin posttranslational regulation. 
a

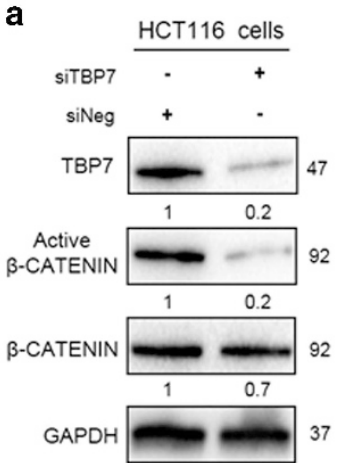

c

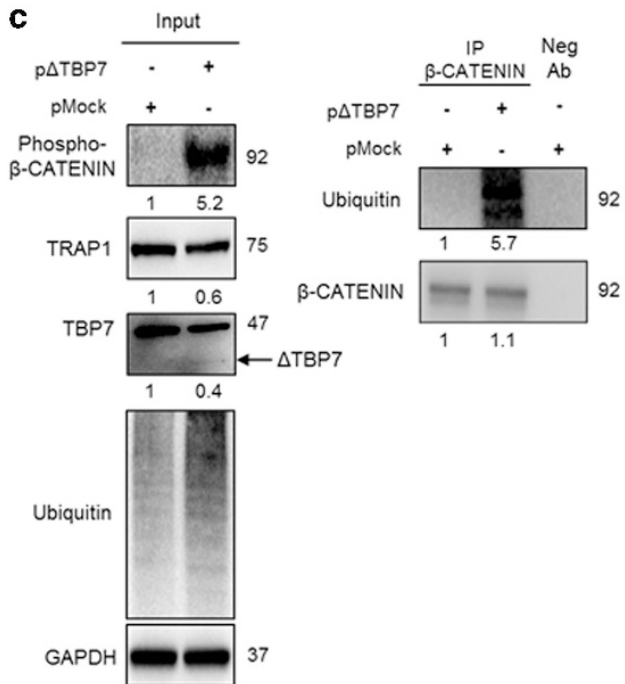

b

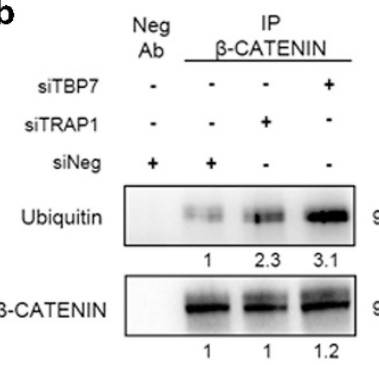

d

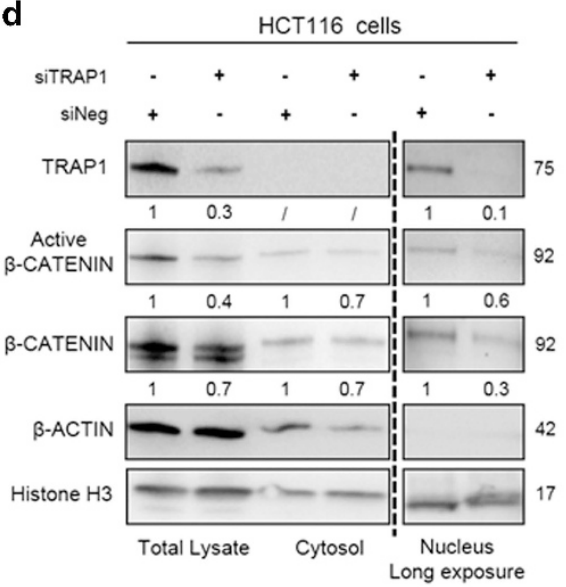

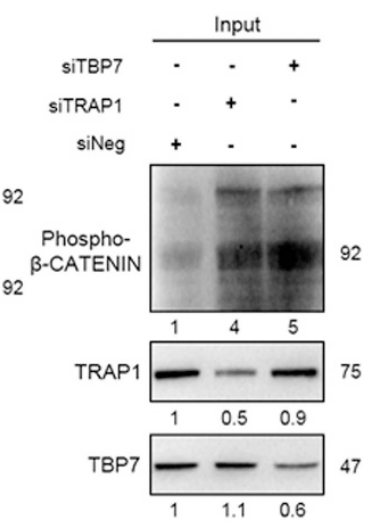

92

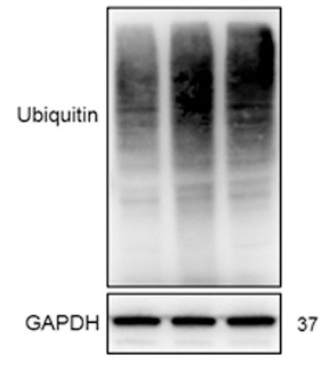

Figure 6 TRAP1 is responsible for regulation of $\beta$-catenin ubiquitination/phosphorylation. (a) Total lysates from HCT116 cells, transfected with control or TBP7 siRNAs, were separated by SDS-PAGE and immunoblotted with indicated antibodies. (b, $\mathbf{c}) \beta$-catenin immunoprecipitates were obtained from HCT116 cells transfected with TRAP1- or TBP7 siRNAs (b) or with a TBP7 delection mutant (c). Cells were incubated with $10 \mathrm{mmol} / \mathrm{l} \mathrm{MG132}$ for $2 \mathrm{~h}$ before cell lysis, separated by SDS-PAGE and immunoblotted with the indicated antibodies. Densitometric band intensities represent ratios between ubiquitinated and total $\beta$-catenin bands in IPs. Neg Ab, total cellular extracts incubated with nonspecific antibody. (c) The arrow indicates the TBP7 deletion mutant band. (d) Total lysates and cytosolic and nuclear fractions were obtained from HCT116 cells transiently silenced for TRAP1 by siRNAs. Equal amounts of proteins were separated by SDS-PAGE and immunoblotted with indicated antibodies

TRAP1 is co-expressed with $\beta$-catenin and Wnt/ $\beta$-catenin-related genes in human CRCs. The relevance of TRAP1 regulation of $\beta$-catenin was further evaluated in human CRCs: $\beta$-catenin protein expression was assessed in 59 CRCs characterized for TRAP1 expression and subdivided in TRAP1-positive (33 tumors) and TRAP1-negative (26 tumors; Figure $8 b)$. Interestingly, a statistically significant coexpression between the two proteins was observed $\left(x^{2}\right.$-test, $P<0.0001$; Figure $8 \mathrm{c}$ and Supplementary Table S3), thus suggesting that TRAP1 quality control on $\beta$-catenin is conserved in human CRCs. In order to support further the hypothesis that TRAP1 regulation of $\mathrm{Wnt} / \beta$-catenin signaling is relevant in vivo, we analyzed two published gene expression data sets (GSE14333, GSE13294) derived from 387 primary resected CRCs. ${ }^{24}$ The Spearman Rank test showed a statistically significant correlation between TRAP1 mRNA levels and the expression of several $\mathrm{Wnt} / \beta$-catenin-related genes (Figure 8d). These data support the notion that TRAP1 control on $W n t / \beta$-catenin signaling pathway is relevant in human colorectal carcinogenesis.

\section{Discussion}

The activation of molecular pathways responsible for protection from environmental stress and regulation of cell biogenesis in response to extracellular stimuli is crucial for the balance between self-renewal, stemness maintenance and differentiation. Intriguingly, this ability of CSCs to adapt to extracellular stressors represents the molecular basis for their intrinsic resistance to cancer therapies and, thus, for tumor repopulation upon initial response to treatments. ${ }^{3}$ Crucial are, in this process, stem cell mitochondria that have important roles in regulating the balance between stemness, differentiation and survival, through a number of mechanisms, including the modulation of oxidative stress, apoptosis and biogenesis. ${ }^{30,31}$ In this perspective, the hypothesis that the mitochondrial molecular chaperone TRAP1 is involved in regulating CSC fate was tested in human CRC, based on its well-established upregulation ${ }^{10}$ and its prognostic role ${ }^{19}$ in this malignancy. Indeed, our data provide, to our knowledge, the first evidence that (i) TRAP1 is preferentially expressed by intestine stem cells and CSCs isolated from CRC cells, (ii) its 
a

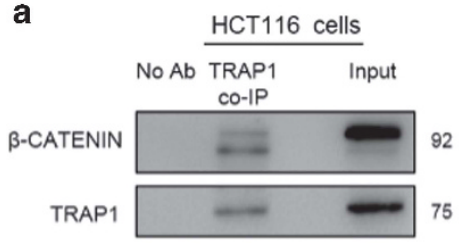

b

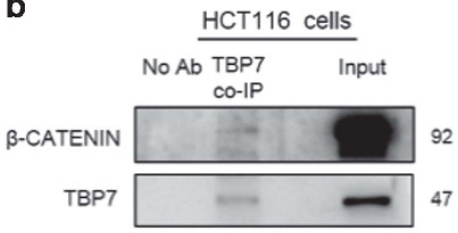

C

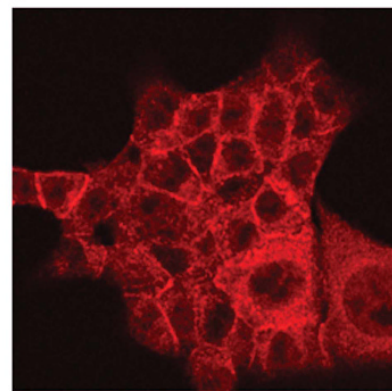

$\beta$-CATENIN

d

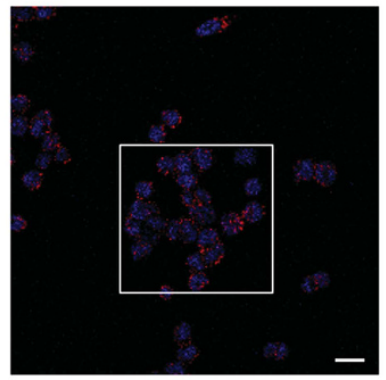

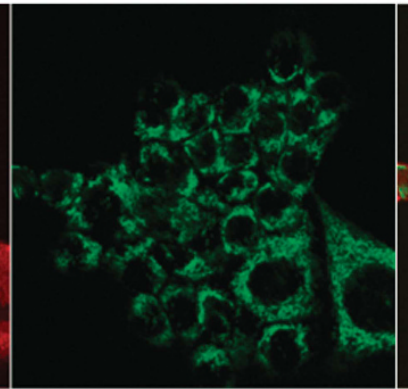

TRAP1

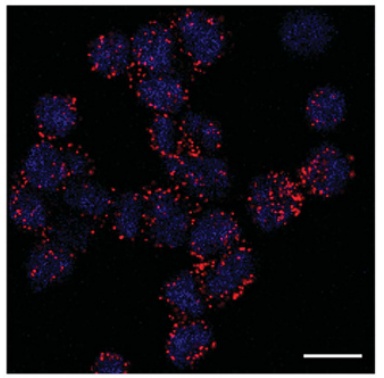

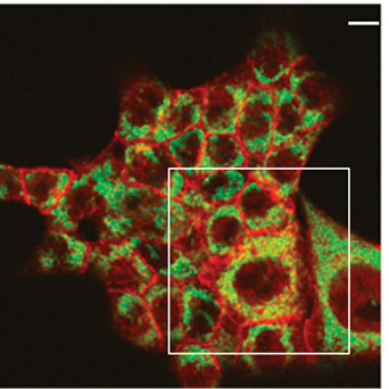

MERGE

e
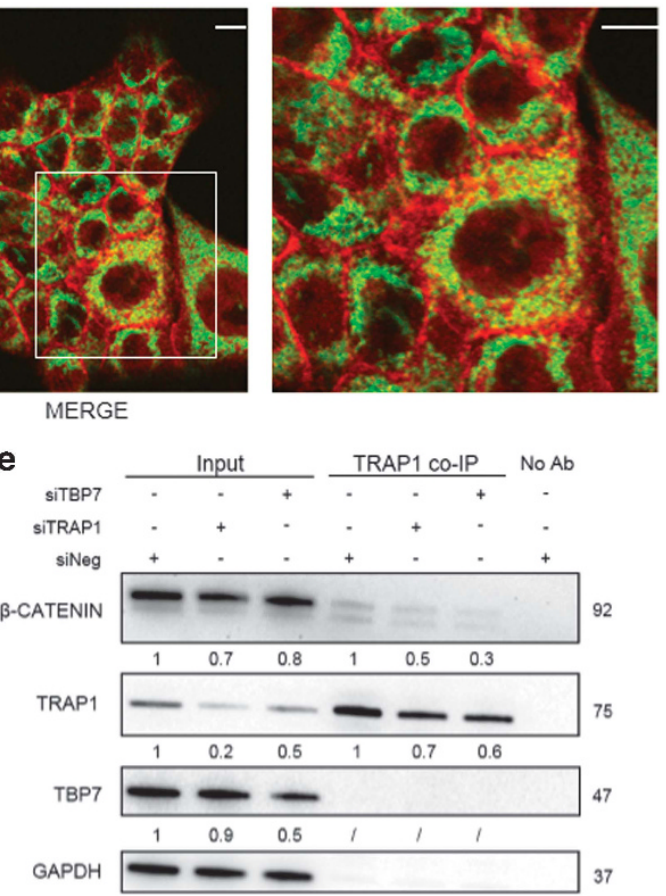

Figure 7 TRAP1 and $\beta$-catenin are components of an intracellular protein complex. (a, b) TRAP1 (a) and TBP7 (b) immunoprecipitates were obtained from HCT116 cells, separated by SDS-PAGE and immunoblotted with the indicated antibodies. (c) Representative immunofluorescence images (left panels) and close-up (right panels) showing colocalization of $\beta$-catenin (red) with TRAP1 (green). Scale bar, $20 \mu \mathrm{m}$. (d) Representative fluorescence image (left panel) and close-up (right panel) showing proximity ligation assay signals (red), detected after labeling HCT116 cells with anti-TRAP1 and anti- $\beta$-catenin antibodies. Nuclei were DAPI-labeled (blue). Scale bar, $20 \mu \mathrm{m}$. (e) TRAP1 immunoprecipitates were obtained from HCT116 cells transfected with control, TRAP1 or TBP7 siRNAs, separated by SDS-PAGE and immunoblotted with the indicated antibodies. A, B and E. No Ab, Total cellular extracts loaded on Control Agarose Resin without antibody

expression enhances the anchorage-independent growth of CRC cells and (iii) its knocking down induces the loss of the stem-like signature with gain of a more-differentiated phenotype. Intriguingly, several features about TRAP1 role in cancer are consistent with this novel function in maintaining stemness. Indeed, TRAP1 has a prevalent mitochondrial localization ${ }^{8,16}$ and is responsible for mitochondrial integrity and regulation of the apoptotic threshold of cancer cells, thereby, favoring drug resistance. ${ }^{8,32}$ This TRAP1 cytoprotective function is particularly relevant in adaptive responses of cancer cells against different stress conditions, including oxidative stress. ${ }^{33}$ Furthermore, TRAP1 regulates organelle-compartmentalized protein folding, especially in cancer cells with high demand for mitochondrial biogenesis and energy production ${ }^{34}$ and is relevant in the cross-talk between mitochondria and endoplasmic reticulum (ER), thus protecting from ER stress. ${ }^{34}$ In such a context, ER-associated TRAP1 regulates proteostasis, having a co-translational quality control on selective client proteins, most of them key genes involved in cancer progression. ${ }^{16,20,26}$ Finally, TRAP1 modulates cancer cell metabolism, favoring the switch from oxidative phosphorylation to aerobic glycolysis. ${ }^{13,14}$
Therefore, based on the knowledge that CSCs are functionally protected in a hypoxic tissue stem cell niche, ${ }^{7}$ the above TRAP1 mitochondrial and extra-mitochondrial functions suggest that its preferential expression in CSCs may be relevant in the regulation of their plasticity, thus favoring protection from stress, protein homeostasis and Warburg metabolism. Although some of these issues deserve further investigation, they are consistent with the evidence that TRAP1 is upregulated in chemo-resistant cancer cells, ${ }^{10,11,20}$ a tumor subpopulation characterized by a stem-like phenotype, self-renewal, tumor initiating capacity and resistance to apoptosis. ${ }^{4}$

Clinically relevant is the observation that TRAP1 is coexpressed with stem cell markers and $W n t / \beta$-catenin-related genes. Indeed, CD44 is a Wnt target gene, promoting cell proliferation $^{35}$ and its mRNA levels, together with CD133 levels, were correlated with synchronous liver metastases in human $\mathrm{CRC}$. $^{36} \mathrm{CD} 166$ is upregulated in human CRCs and its expression correlates with shortened patient survival. ${ }^{37}$ In such a context, TRAP1 is upregulated in $\sim 60 \%$ of human $\mathrm{CRCs}^{10}$ and its upregulation is associated with enhanced lymph node metastases and shorter overall survival. ${ }^{19,38}$ 
a

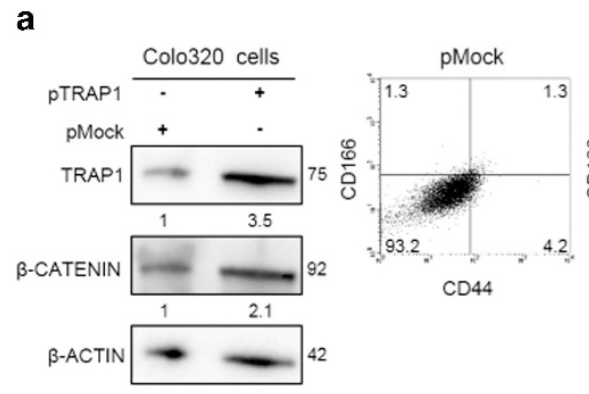

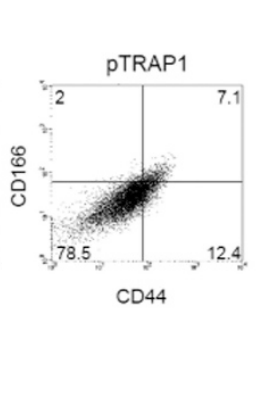

c

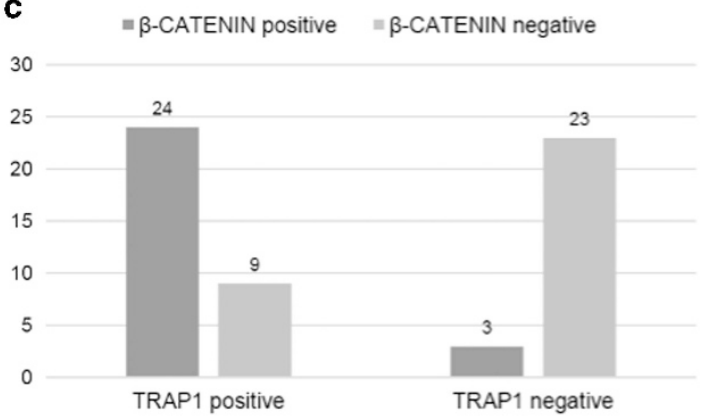

b

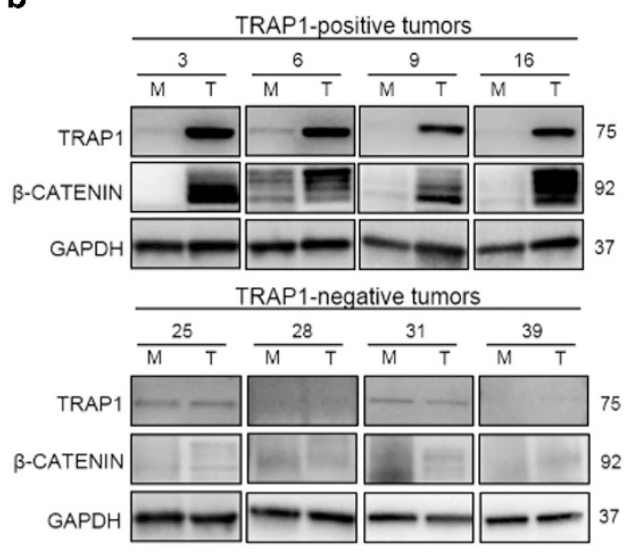

d
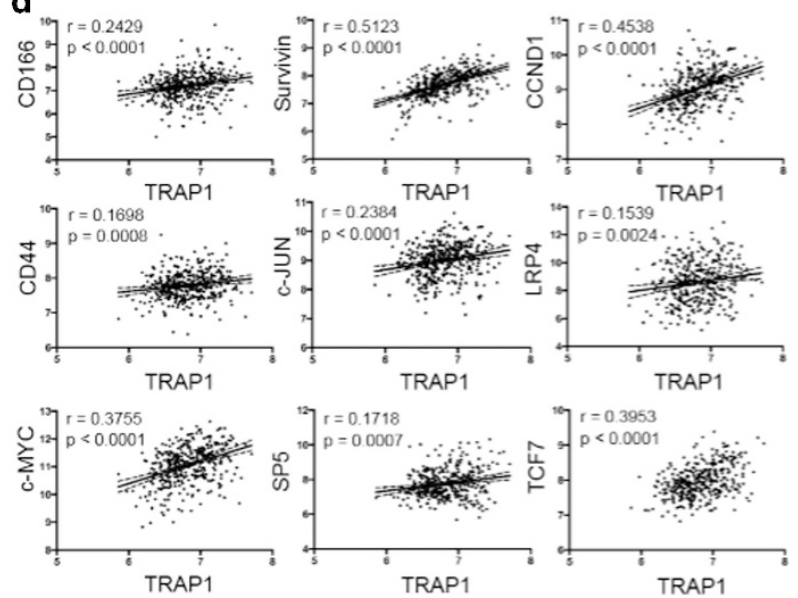

Figure 8 TRAP1 is co-expressed with $\beta$-catenin and Wnt $\beta$-catenin-related genes in human colorectal carcinomas. (a) TRAP1 and $\beta$-catenin immunoblot analysis (left panel) and CD166/CD44 expression levels in HCT116 cells transfected with pMock and pTRAP1. (b) Total cell lysates from eight representative human CRCs (T) and respective peritumoral non-infiltrated mucosas (M) were separated by SDS-PAGE and immunoblotted with the indicated antibodies. (c) Distribution of TRAP1 and $\beta$-catenin protein levels in our series of 59 colorectal carcinomas $(P<0.0001)$. (d) Dot plots representing the correlation between TRAP1 and specific Wnt/ $\beta$-catenin target genes expression levels obtained from two public data sets of 387 human CRCs

Thus, the co-expression of TRAP1 and stem cell markers observed in vitro and in vivo and the gene expression profiling data, which suggest that TRAP1 silencing favors the shift from the Stem-like to the more-differentiated Enterocyte phenotype, support the hypothesis that TRAP1 is responsible for the Stemlike phenotype of CRCs, a subtype with poor prognosis. ${ }^{24}$

Finally, our data suggest that TRAP1 maintenance of stemness is mediated by its regulation on $\mathrm{Wnt} / \beta$-catenin pathway, and this likely occurs through the modulation of the expression of specific frizzled receptor ligands and the regulation of $\beta$-catenin ubiquitination/phosphorylation. Indeed, $\beta$-catenin is an intracellular signal transducer in Wnt signaling pathway and acts as a transcriptional factor upon translocation into the nucleus. ${ }^{27}$ In physiological conditions, Wnt $\beta$-catenin activity depends on the amount of $\beta$-catenin located in the cytoplasm, and in the absence of a Wnt ligand, a 'destruction complex' phosphorylates cytosolic $\beta$-catenin, which is subsequently degraded by the ubiquitin-proteasome system. ${ }^{29}$ By contrast, $\mathrm{Wnt} / \beta$-catenin signaling is constitutively active in $\sim 90 \%$ of $\mathrm{CRCs},{ }^{27}$ and $\beta$-catenin is the main regulatory pathway of stemness in this malignancy. ${ }^{29}$ It is, thus, intriguing that (i) TRAP1 interacts with $\beta$-catenin and both proteins are components of an intracellular protein complex, (ii) high levels of $\beta$-catenin are present in TRAP1-rich CRC cell lines and human CRCs, (iii) TRAP1 is co-expressed with several Wnt/ $\beta$ - catenin target genes in human CRCs. By contrast, $\beta$-catenin phosphorylation and ubiquitination are enhanced upon suppression of TRAP1 network, this resulting in reduced nuclear $\beta$-catenin and downregulation of a number of Wnt target genes. These observations provide the first evidence that TRAP1 network regulates $\mathrm{Wnt} / \beta$-catenin pathway and that this regulation occurs at level of $\beta$-catenin ubiquitination and phosphorylation, a process involved in the modulation of $\beta$-catenin degradation. ${ }^{29}$ This observation is consistent with the general view that TRAP1, together with the proteasome regulatory protein TBP7, exerts a quality-control regulation on specific client proteins. ${ }^{20,26}$ In such a context, the evidence that the disruption of TRAP1/TBP7 quality-control network enhances the phosphorylation and the ubiquitination of $\beta$ catenin and that $\beta$-catenin interacts with both TRAP1 and TBP7 suggests that TRAP1/TBP7 regulation of $\beta$-catenin ubiquitination/phosphorylation occurs in close association with the $\beta$-catenin degradation pathway. Intriguingly, this hypothesis provides the molecular basis to decipher the mechanism used by TRAP1 in maintenance of stemness, and further highlights the relevance of TRAP1 quality-control network in colon carcinogenesis. Indeed, we recently reported that (i) TRAP1 regulates BRAF synthesis and modulates its downstream pathway in CRC cells, ${ }^{26}$ (ii) BRAF phosphorylates TRAP1 to enhance the apoptotic threshold of cancer 
cells $^{39}$ and (iii) TRAP1 targeting is a valuable strategy to target BRAF-driven human CRC cells. ${ }^{26,39}$ Taken in a whole, these observations indicate that TRAP1 upregulation is a key event in colorectal tumorigenesis and support the concept that TRAP1 inhibition deserves to be evaluated as a strategy to target CRC cells with stem cell traits.

\begin{abstract}
Materials and Methods
Cell lines and constructs. CRC k-ras G13D HCT116, b-raf V600E HT29, ras and raf wild-type COLO320 and $\mathrm{CaC} 22$ cells were purchased from American Type Culture Collection (ATCC, Manassas, VA, USA). Cell lines were routinely monitored in our laboratory by microscopic morphology, whereas cell line authentication was verified by STR profiling in July 2013, according to ATCC product description. HT29, CaCo2, COLO320 cells were cultured in DMEM supplemented with $10 \%$ (v/v) fetal bovine serum (FBS), $1.5 \mathrm{mM}$ glutamine and $100 \mathrm{U} / \mathrm{ml}$ penicillin and streptomycin, HCT116 cells in McCoy's medium supplemented with $10 \%(\mathrm{v} / \mathrm{v}) \mathrm{FBS}, 0.75 \mathrm{mM}$ glutamine and $10 \mathrm{U} / \mathrm{ml}$ penicillin and streptomycin. TRAP1 stably interfered HCT116 cells, TRAP1 transient silencing, full-length TRAP1 transfection and the TBP7 delection mutant, $\triangle$ TBP7-FLAG construct, were obtained as previously described. ${ }^{12,16-18}$ Two independent siRNAs of TRAP1 were used (Qiagen, Hilden, Germany, Cat. no. SI00115150 and no. SI00115164). Unless otherwise specified, reagents were purchased from SigmaAldrich (Saint Louis, MO, USA).
\end{abstract}

Flow cytometry and cell sorting. Cell suspensions were reconstituted to a final concentration of $1.0 \times 10^{6} \mathrm{cells} / \mathrm{ml}$ in phosphate buffer saline (PBS) containing $0.1 \%(\mathrm{w} / \mathrm{v}) \mathrm{NaN}_{3}$ and $5 \%(\mathrm{v} / \mathrm{v}) \mathrm{FBS}$. As a preliminary step, intrinsic cell fluorescence was analyzed, using unlabeled samples. For specific staining, $100 \mu \mathrm{l}$ of each cell suspension were incubated with $10 \mu \mathrm{l}$ of each primary antibody for $20 \mathrm{~min}$ at $4{ }^{\circ} \mathrm{C}$. The following primary antibodies were used: mouse monoclonal anti-CD166 (PE) (555749) and mouse monoclonal anti-CD44 (APC) (559942) from Becton Dickinson (San Jose, CA, USA), rabbit polyclonal anti-TRAP1 (FITC) (ABIN686654) from Antibodies-online $\mathrm{GmbH}$ (Aachen, Germany), mouse monoclonal anti-CD133/1 (PE) (130-080-801) from Miltenyi Biotec (Bergisch Gladbach, Germany). For TRAP1 staining, given its predominant mitochondrial localization, cells were permeabilized with Fix \& Perm Kit (Life Technologies, Carlsbad, CA, USA), before incubation with anti-TRAP1 antibody. Cells were finally analyzed by flow cytometry with FACSCalibur (Becton Dickinson, Franklin Lakes, NJ, USA). For cell sorting, cells were incubated with anti-CD166 or anti-TRAP1 antibody for $20 \mathrm{~min}$ at $4{ }^{\circ} \mathrm{C}$. After washing with PBS, cells were FACS sorted with a MoFlo Astrios (Beckman Coulter, Brea, CA, USA).

Colony-forming assay. In total, $1.25 \times 10^{4}$ cells were suspended in prewarmed $\left(40^{\circ} \mathrm{C}\right) \quad 0.7 \%(\mathrm{w} / \mathrm{v})$ agarose solution containing $10 \%(\mathrm{v} / \mathrm{v})$ FBS DMEM, seeded on the top of a bottom layer of a $0.8 \%(\mathrm{w} / \mathrm{v})$ agar gel containing $10 \%(\mathrm{v} / \mathrm{v})$ FBS DMEM, and left growing for 15 days with medium changes every 3 days. Finally, colonies were fixed with methanol/acetic acid solution (1:7) and colored with crystal violet. ${ }^{39}$ Density of transformation colonies were compared by cell counts and represented as mean \pm S.D.

Real-time RT-PCR analysis. Total RNA was extracted from cell pellets using the TRIzol Reagent (Life Technologies). For first strand synthesis of cDNA, $1 \mu \mathrm{g}$ of RNA was used in a 20- $\mu$ l reaction mixture utilizing a Transcriptor First Strand cDNA Synthesis Kit (Roche, Mannheim, Germany). For Real-time PCR analysis, $0.5 \mathrm{ng}$ of cDNA samples were amplified using the LightCycler 480 SYBR Green I Master (Roche) in a LightCycler 480 (Roche). The following primers were used: WNT3A forward 5'-CTGCAGGAACTACGTGGAGATC-3', reverse 5'-GCAATGGCGTGGAC AAAG-3' (PCR product $183 \mathrm{bp);} \mathrm{WNT16} \mathrm{forward} \mathrm{5'-CTTTGGCTACGAGCTGA}$ GC-3', reverse 5'-CATGCCATACTGGACATCATC-3' (PCR product 205 bp); c-MYC forward 5'-TTCGGGTAGTGGAAAACCAG-3', reverse 5'-CAGCAGCTCGA ATTTCTTCC-3' (PCR product 203 bp); CCND1 forward 5'-CTACTACCGCCT CACACGCTT-3', reverse 5'-AGCCCTGGAGTCAAGCC-3' (PCR product 198 bp); c-JUN forward 5'-CTACGCAAACCTCAGCAACTTC-3', reverse 5'-GTCT GAGGCTCCTCCTTCAG-3' (PCR product $186 \mathrm{bp}$ ); CTNNB1 forward 5'-GCA ATGACTCGAGCTCAGAG-3', reverse 5'-GCAAGTTCTGCATCATCTTG-3' (PCR product $188 \mathrm{bp}$ ); NKD1 forward 5'-TGTATGACTTTGACAACAACG-3', reverse $5^{\prime}$-TGATTGA
CAAGGACGCTC-3' (PCR product $181 \mathrm{bp}) ; \quad$ SP5 forward $5^{\prime}$-TTTCTC CAGGACCGCAC-3', reverse 5'-GAAGAGCCTGGAGGGTGA-3' (PCR product 168 bp); AXIN2 forward 5'-AGGTTCTGGCTATGTCTTTG-3', reverse 5'-AAATG AGGTAGAGACACTTGG-3' (PCR product $201 \mathrm{bp}$ ); TRAP1 forward 5'-CGC AGCATCTTCTACGTGC-3', reverse 5'-CTGATGAGTGCGCTCTCC-3' (PCR product $200 \mathrm{bp}) ; \beta$-ACTIN forward $5^{\prime}$-CGCAAAGACCTGTACGC- $3^{\prime}$, reverse $5^{\prime}$-CACA CGGAGTACTTGCGC-3' (PCR product 152 bp). Primers were designed to be intron spanning. PCR reaction conditions were as follows: pre-incubation at $95^{\circ} \mathrm{C}$ for $5 \mathrm{~min}$, followed by $45 \mathrm{cycles}$ of $10 \mathrm{~s}$ at $95^{\circ} \mathrm{C}, 10 \mathrm{~s}$ at $60^{\circ} \mathrm{C}, 10 \mathrm{~s}$ at $72{ }^{\circ} \mathrm{C}$. $\beta$-ACTIN was chosen as an internal control.

Immunoblot analysis. Total cell lysates were obtained by homogenization of cell pellets and tissue samples in a cold lysis buffer $(20 \mathrm{mM}$ Tris, pH 7.5 containing $300 \mathrm{mM}$ sucrose, $60 \mathrm{mM} \mathrm{KCl}, 15 \mathrm{mM} \mathrm{NaCl}, 5 \%$ (v/v) glycerol, $2 \mathrm{mM}$ EDTA, $1 \%$ (v/v) Triton X-100, $1 \mathrm{mM}$ PMSF, $2 \mathrm{mg} / \mathrm{ml}$ aprotinin, $2 \mathrm{mg} / \mathrm{ml}$ leupetin and $0.2 \%$ (w/v) deoxycholate) for $2 \mathrm{~min}$ at $4{ }^{\circ} \mathrm{C}$ and further sonication for $30 \mathrm{~s}$ on ice. Immunoblot analysis was performed as previously reported..$^{20}$ Cytosolic and nuclear fractions were purified by the Qproteome Mitochondria Isolation kit (Qiagen Venlo, Limburgo, the Netherlands) according to the manufacturer's protocol. Protein immunoprecipitation was carried out starting from $1 \mathrm{mg}$ of total protein extracts. Lysates were pre-cleared by incubating with protein A/G-Agarose (Santa Cruz Biotechnologies, Santa Cruz, CA, USA) for $1 \mathrm{~h}$ at $4{ }^{\circ} \mathrm{C}$ and then incubated with gentle shaking for $18 \mathrm{~h}$ at $4{ }^{\circ} \mathrm{C}$ with specific antibodies, after which samples were further incubated for $1 \mathrm{~h}$ at $4{ }^{\circ} \mathrm{C}$ with fresh beads. Beads were collected by centrifugation and washed twice in lysis buffer. Co-ip experiments were carried out by using Pierce CO-Immunoprecipitation kit (Thermo Scientific, Waltham, MA, USA) by covalently coupling antibodies onto an amine-reactive resin, according to the manufacturer's instructions. The following antibodies were used: mouse monoclonal anti-HSP75 (sc-73604), mouse monoclonal anti-PSMC4 (TBP7) (sc-166003), mouse monoclonal anti-Ubiquitin (sc-8017), mouse monoclonal anti-GAPDH (sc-47724) and mouse monoclonal anti- $\beta$-Actin (sc-47778) from Santa Cruz Biotechnology, rabbit monoclonal anti-Histone H3 (\#4499), rabbit monoclonal anti-active- $\beta$-catenin (\#8814), rabbit polyclonal anti-phospho- $\beta$-catenin (\#9561), and rabbit polyclonal anti- $\beta$-catenin (\#9562), rabbit monoclonal anti-Survivin (\#2808) from Cell Signaling Technology (Boston, MA, USA), rabbit monoclonal anti-WNT3A (ab172612) from Abcam (Cambridge, UK), rabbit polyclonal anti-Survivin (NB 500-201H) from Novus Biologicals (Littleton, CO, USA). Protein levels were quantified by densitometric analysis using the ImageJ software and normalized according to the expression of the housekeeping gene.

Immunohistochemistry. Normal colon tissues were obtained from surgical intestinal excisions for benign pathology. Five- $\mu \mathrm{m}$ serial sections from formalin-fixed and paraffin-embedded blocks were cut and mounted on poly-L-lysine-coated glass slides. Immunohistochemical analysis was performed using a Benchmark autostainer (Ventana Medical Systems, Tucson, AZ, USA). Sections were incubated with mouse monoclonal anti-TRAP1 (sc-73604, Santa Cruz Biotechnology) and anti-CD166 (sc-74558, Santa Cruz Biotechnology) antibodies (1:750 dilution). Negative controls were performed omitting primary antibody. Sections were counterstained with type-II-Gill's haematoxylin, dehydrated with ethanol and permanently coverslipped. The sections were scanned at high magnification to assess the positivity of staining.

Confocal microscopy and proximity ligation assay. Cells were seeded on coverslips and, after $48 \mathrm{~h}$, fixed with $4 \%$ (w/v) paraformaldehyde in PBS for 20 min. Cells were blocked and permeabilized with $0.4 \%$ (w/v) BSA, $0.1 \%(\mathrm{v} / \mathrm{v})$ Triton X-100, 5\% (v/v) FBS in PBS for $15 \mathrm{~min}$ at RT before staining over night with indicated primary antibodies. For colocalization experiments, the corresponding secondary Cy3/Cy5-conjugated antibodies were used, whereas for proximity ligation assay, the Duolink In Situ Red Starter Kit Mouse/Rabbit from Sigma-Aldrich (DU092101) was used, according to the manufacturer's instructions. Image acquisition was performed by confocal laser-scanning microscopy using Zeiss 510 LSM from Carl Zeiss Microimaging (Oberkochen, Germany).

Colon carcinoma cell lines subclassification. CRC subtype signature reported by Sadanandam et al. (Supplementary Tables S1 and S2) ${ }^{24}$ was used for this analysis. This signature, defined by a list of 758 differentially expressed genes (up, down or unchanged), produced five colorectal cancer subtypes, that is, Inflammatory, Goblet-like, Enterocyte, TA and Stem-like. The concordance between CRC subtype signatures and the previously reported expression profile of shTRAP1 
HCT 116 (ref. 26) was calculated. To this purpose, logFC of these data was used to generate a heat map with Euclidean distance and average linkage method. Furthermore, we defined a cutoff of $\log \mathrm{FC} \pm 0.1$ to define differentially regulated genes. Genes with a logFC $>0.1$ or $<-0.1$ were, respectively, classified as upor downregulated, whereas all other genes were classified as unchanged (Supplementary Table S1). Genes that agreed for being up, down or unchanged compared to CRC subtype signatures were defined as concordant genes, all the others were defined as discordant (Supplementary Table S2).

Tumor specimens. Specimens from 59 human CRCs and corresponding normal, non-infiltrated, peritumoral mucosa were obtained from the General Surgery Unit of the University of Foggia. Express written informed consent to use biologic specimens for investigational procedures was obtained from all patients. In order to compare levels of TRAP1 and $\beta$-catenin in different tumor specimens, protein levels were quantified by densitometric analysis using the Quantity One 4.5.0 software (BioRad Laboratories GmbH, Hercules, CA, USA) and expressed as time increase/ decrease in tumors compared with the respective peritumoral non-infiltrated mucosa (Supplementary Table S3). TRAP1 expression levels were regarded as being upregulated if they had increased at least threefold in comparison to the corresponding non-infiltrated peritumoral mucosa, whereas $\beta$-catenin levels were regarded as upregulated if they had increased at least twofold compared to the corresponding non-infiltrated peritumoral mucosa.

Co-expression between TRAP1 and Wnt/ $\beta$-catenin target genes in human CRCs. Public data sets (GSE13294 and GSE14333) were used to analyze the co-expression between TRAP1 and selected genes responsible for the Stem-like signature or target genes of Wnt $\beta$-catenin pathway. Data sets were preprocessed and combined using RMA method (bioconductor library; affy) as reported by Sadanandam et al..$^{24}$ (http://www.nature.com/protocolexchange/protocols/2053\#/ procedure). RMA processed gene expression profile data were further analyzed to establish the co-expression with TRAP1 mRNA levels. Spearman rank-order correlation method was used to calculate correlation coefficients and $P$-values. Results are graphically reported as dot plots with regression lines.

Statistical analysis. Two-sided ANOVA test was used to establish statistical differences in antigen expression between CRC cell lines; two-sided paired $t$-test to establish statistical differences in gene expression between TRAP1-modulated and control cells; $\chi^{2}$-test to establish statistical significance of TRAP1 and $\beta$-catenin coexpression in human CRCs; Spearman rank-order correlation method to calculate correlation coefficients and $P$-values of TRAP1 and $\mathrm{Wnt} / \beta$-catenin genes. Statistically significant values are reported in Figure Legends. All experiments were independently performed at least three times and three technical replicates were used for statistical analysis. Data represent mean+S.D.

\section{Conflict of Interest}

The authors declare no conflict of interest.

Acknowledgements. This work was supported by AIRC (Grant IG2015 Id. 16738 to ML and FE), the Italian Ministry of Health (Grant GR-2010-2310057 to FM) and the University of Foggia (PRA Grant to ML).

1. Shen N, Liu C, Li J, Chen X, Yang Y, Zhu $Y$ et al. A phosphorylation-related variant ADD1-rs4963 modifies the risk of colorectal cancer. PLoS One 2015; 10: e0121485.

2. Hoekstra E, Kodach LL, Das AM, Ruela-de-Sousa RR, Ferreira CV, Hardwick JC et al. Low molecular weight protein tyrosine phosphatase (LMWPTP) upregulation mediates malignant potential in colorectal cancer. Oncotarget 2015; 6: 8300-8312.

3. Colak S, Medema JP. Cancer stem cells-important players in tumor therapy resistance. FEBS J 2014; 281: 4779-4791.

4. Nguyen LV, Vanner R, Dirks $P$, Eaves CJ. Cancer stem cells: an evolving concept. Nat Rev Cancer 2012; 12: 133-143.

5. Reya T, Morrison SJ, Clarke MF, Weissman IL. Stem cells, cancer, and cancer stem cells. Nature 2001; 414: 105-111.

6. Ito K, Suda T. Metabolic requirements for the maintenance of self-renewing stem cells. Nat Rev Mol Cell Biol 2014; 15: 243-256.

7. Plaks V, Kong N, Werb Z. The cancer stem cell niche: how essential is the niche in regulating stemness of tumor cells? Cell Stem Cell 2015; 16: 225-238.
8. Kang BH, Plescia J, Dohi T, Rosa J, Doxsey SJ, Altieri DC. Regulation of tumor cell mitochondrial homeostasis by an organelle-specific Hsp90 chaperone network. Cell 2007; 131: 257-270.

9. Amoroso MR, Matassa DS, Sisinni L, Lettini G, Landriscina M, Esposito F. TRAP1 revisited: novel localizations and functions of a 'next-generation' biomarker (review). Int J Oncol 2014; 45: 969-977.

10. Costantino E, Maddalena F, Calise S, Piscazzi A, Tirino V, Fersini A et al. TRAP1, a novel mitochondrial chaperone responsible for multi-drug resistance and protection from apoptotis in human colorectal carcinoma cells. Cancer Lett 2009; 279: 39-46.

11. Maddalena F, Sisinni L, Lettini G, Condelli V, Matassa DS, Piscazzi A et al. Resistance to paclitxel in breast carcinoma cells requires a quality control of mitochondrial antiapoptotic proteins by TRAP1. Mol Oncol 2013; 7: 895-906.

12. Sisinni L, Maddalena F, Lettini G, Condelli V, Matassa DS, Esposito F et al. TRAP1 role in endoplasmic reticulum stress protection favors resistance to anthracyclins in breast carcinoma cells. Int J Oncol 2014; 44: 573-582.

13. Sciacovelli M, Guzzo G, Morello V, Frezza C, Zheng L, Nannini N et al. The mitochondrial chaperone TRAP1 promotes neoplastic growth by inhibiting succinate dehydrogenase. Cell Metab 2013; 17: 988-999.

14. Yoshida S, Tsutsumi S, Muhlebach G, Sourbier C, Lee MJ, Lee S et al. Molecular chaperone TRAP1 regulates a metabolic switch between mitochondrial respiration and aerobic glycolysis. Proc Natl Acad Sci USA 2013; 110: E1604-E1612.

15. Chae YC, Angelin A, Lisanti S, Kossenkov AV, Speicher KD, Wang H et al. Landscape of the mitochondrial Hsp90 metabolome in tumours. Nat Commun 2013; 4: 2139.

16. Amoroso MR, Matassa DS, Laudiero G, Egorova AV, Polishchuk RS, Maddalena F et al. TRAP1 and the proteasome regulatory particle TBP7/Rpt3 interact in the endoplasmic reticulum and control cellular ubiquitination of specific mitochondrial proteins. Cell Death Differ 2012; 19: 592-604

17. Matassa DS, Amoroso MR, Agliarulo I, Maddalena F, Sisinni L, Paladino S et al. Translational control in the stress adaptive response of cancer cells: a novel role for the heat shock protein TRAP1. Cell Death Dis 2013; 4: e851.

18. Matassa DS, Agliarulo I, Amoroso MR, Maddalena F, Sepe L, Ferrari MC et al. TRAP1dependent regulation of $\mathrm{p70S6 \textrm {K }}$ is involved in the attenuation of protein synthesis and cell migration: relevance in human colorectal tumors. Mol Oncol 2014; 8: 1482-1494.

19. Gao JY, Song BR, Peng JJ, Lu YM. Correlation between mitochondrial TRAP-1 expression and lymph node metastasis in colorectal cancer. World J Gastroenterol 2012; 18: 5965-5971.

20. Landriscina M, Laudiero G, Maddalena F, Amoroso MR, Piscazzi A, Cozzolino F et al. Mitochondrial chaperone Trap1 and the calcium binding protein Sorcin interact and protect cells against apoptosis induced by antiblastic agents. Cancer Res 2010; 70: 6577-6586.

21. Schuijers J, Clevers H. Adult mammalian stem cells: the role of Wnt, Lgr5 and R-spondins. EMBO J 2012; 31: 2685-2696.

22. Dalerba $P$, Dylla SJ, Park IK, Liu R, Wang $X$, Cho RW et al. Phenotypic characterization of human colorectal cancer stem cells. Proc Natl Acad Sci USA 2007; 104: 10158-10163.

23. Kemper K, Grandela C, Medema JP. Molecular identification and targeting of colorectal cancer stem cells. Oncotarget 2010; 1: 387-395.

24. Sadanandam A, Lyssiotis CA, Homicsko K, Collisson EA, Gibb WJ, Wullschleger $S$ et al. A colorectal cancer classification system that associates cellular phenotype and responses to therapy. Nat Med 2013; 19: 619-625.

25. Vermeulen L, De Sousa E, Melo F, van der Heijden M, Cameron K, de Jong JH, Borovski T et al. Wnt activity defines colon cancer stem cells and is regulated by the microenvironment. Nat Cell Biol 2010; 12: 468-476.

26. Condelli V, Piscazzi A, Sisinni L, Matassa DS, Maddalena F, Lettini G et al. TRAP1 is involved in BRAF regulation and downstream attenuation of ERK phosphorylation and cellcycle progression: a novel target for BRAF-mutated colorectal tumors. Cancer Res 2014; 74: 6693-6704.

27. Takebe N, Miele L, Harris PJ, Jeong W, Bando H, Kahn M et al. Targeting Notch, Hedgehog, and Wnt pathways in cancer stem cells: clinical update. Nat Rev Clin Oncol 2015; 12: 445-464.

28. Herbst A, Jurinovic V, Krebs S. Thieme SE, Blum H, Göke B et al. Comprehensive analysis of $\beta$-catenin target genes in colorectal carcinoma cell lines with deregulated Wnt $/ \beta$-catenin signaling. BMC Genomics 2014; 15: 74

29. Voloshanenko O, Erdmann G, Dubash TD, Augustin I, Metzig M, Moffa G et al. Wnt secretion is required to maintain high levels of Wnt activity in colon cancer cells. Nat Commun 2013; 4: 2610.

30. Kadye R, Kramer AH, Joos-Vandewalle J, Parsons M, Njengele Z, Hoppe H et al. Guardian of the furnace: mitochondria, TRAP1, ROS and stem cell maintenance. IUBMB Life 2014; 66: 42-45.

31. Song IS, Jeong JY, Jeong SH, Kim HK, Ko KS, Rhee BD et al. Mitochondria as therapeutic targets for cancer stem cells. World J Stem Cells 2015; 7: 418-427.

32. Montesano Gesualdi N, Chirico G, Pirozzi G, Costantino E, Landriscina M, Esposito F et al. Tumor necrosis factor-associated protein 1 (TRAP-1) protects cells from oxidative stress and apoptosis. Stress 2007; 10: 342-350.

33. Hua G, Zhang $Q$, Fan $Z$. Heat shock protein 75 (TRAP1) antagonizes reactive oxygen species generation and protects cells from granzyme M-mediated apoptosis. J Biol Chem 2007; 282: 20553-20560.

34. Siegelin MD, Dohi T, Raskett CM, Orlowski GM, Powers CM, Gilbert CA et al. Exploiting the mitochondrial unfolded protein response for cancer therapy in mice and human cells. J Clin Invest 2011; 121: 1349-1360.

35. Du L, Wang H, He L, Zhang J, Ni B, Wang X et al. CD44 is of functional importance for colorectal cancer stem cells. Clin Cancer Res 2008; 14: 6751-6760. 
TRAP1 regulates colon cancer stem cell phenotype

G Lettini et al

36. Jing F, Kim HJ, Kim CH, Kim YJ, Lee JH, Kim HR et al. Colon cancer stem cell markers CD44 and CD133 in patients with colorectal cancer and synchronous hepatic metastases. Int J Oncol 2015; 46: 1582-1588.

37. Weichert W, Knösel T, Bellach J, Dietel M, Kristiansen G et al. G. ALCAM/CD166 is overexpressed in colorectal carcinoma and correlates with shortened patient survival. J Clin Pathol 2004; 57: 1160-1164
38. Han JJ, Baek SK, Lee JJ, Kim GY, Kim SY, Lee SH. Combination of TRAP1 and ERCC1 expression predicts clinical outcomes in metastatic colorectal cancer treated with oxaliplatin/ 5-fluorouracil. Cancer Res Treat 2014; 46: 55-64.

39. Condelli V, Maddalena F, Sisinni L, Lettini G, Matassa DS, Piscazzi A et al. Targeting TRAP1 as a downstream effector of BRAF cytoprotective pathway: a novel strategy for human BRAF-driven colorectal carcinoma. Oncotarget 2015; 6: 22298-22309.

Supplementary Information accompanies this paper on Cell Death and Differentiation website (http://www.nature.com/cdd) 